\title{
L'orangé et le bleu chez Cendrars, simultanément
}

Philippe Enrico

Résumé: Lors de leur première rencontre à Paris en 1923, Blaise Cendrars offre à Tarsila do Amaral une petite peinture à l'huile sur carton, intitulée depuis Torre Eiffel, une des rares peintures que Cendrars ait peintes, dix ans auparavant, avec les couleurs de son ami Robert Delaunay. Vingt-deux ans après son dernier séjour au Brésil, Cendrars va dédier à Tarsila le dernier récit de ses mémoires, qui a pour décor la fazenda du "Morro Azul" d'un certain Oswaldo Padroso, l'inventeur d'une nouvelle constellation, la "Tour Eiffel Sidérale", qui va donner son nom au récit. Dans l'ambivalence des contrastes simultanés, orangés de la passion et bleus de l'introspection vont se mêler une vie durant, tout comme aventure et écriture, la braise couvant sous la cendre, tel que le poète nous le chante pour toujours.

Mots-clé: Blaise Cendrars, Tarsila do Amaral, tour Eiffel, contraste simultané, ambivalence. 
Vient de paraître, cette "année de la France au Brésil", la traduction du Lotissement du ciel (O loteamento do céu) de Blaise Cendrars, qui avait été publié la première fois à Paris en 1949 aux éditions Denoël, livre qui, soixante ans après, se trouve embellit sur sa couverture brésilienne de la reproduction d'une toile de Tarsila do Amaral datée de 1924, Carnaval em Madureira, où l'on voit parmi passantes et enfants une tour Eiffel de bois, dressée probablement au milieu d'une place de Madureira, faubourg nord de Rio de Janeiro, pour le Carnaval. Accrochés à son sommet, quelques drapeaux et le petit dirigeable N-3 de Santos Dumont la contournant le 13 novembre 1899, nargant les prévisions astrologiques qui pariaient pour ce jour la fin du monde.

Remontons le cours du temps pour arriver à Paris, rue du MontDore, le 28 mai 1923.

Tarsila do Amaral et Oswald de Andrade visitent pour la première fois Blaise Cendrars chez la mère de Raymone Duchâteau, sa compagne. Cendrars offre à "Madame Tarsila do Amaral" une petite peinture qu'il lui dédicace. Les liens d'amitiés sont alors noués. Tarsila est née un premier septembre, aînée de Cendrars d'un an jour pour jour, d'Oswald, un peu plus. Ils ont plus ou moins l'âge de la Tour Eiffel, dont la construction avait commencé en 1887. L'atelier de Tarsila se trouve à proximité de l'appartement de Madame Duchâteau, près de la place Clichy. Tarsila était venue étudier lors de son précédent séjour, en 1920, aux Académies Julian et Emile Renard, mais cette fois-ci, elle préfère fréquenter les ateliers "modernes" des cubistes Albert Gleizes, André Lhote ou Fernand Léger, l'ami de Cendrars, dont on retrouve les enseignements dans notre Carnaval em Madureira. Place Clichy: le nombril du monde comme la voyait Paulo Prado, un des plus grands producteurs mondiaux de café, francophile particulièrement lettré qui, avec Oswald et leurs amis, allait initier Cendrars aux mystères du Brésil. Et pour commencer ferme, il allait l'y inviter l'année d'après pour la réalisation de

1. Cendrars épousera Raymone vingt-six ans plus tard, en 1949, année de la publication du Lotissement du ciel.

2. "Desde aí ficamos muito amigos", nous dit Tarsila do Amaral. In: EULÁLIO. A aventura brasileira de Blaise Cendrars, p. 264. 
divers projets, dont un film de propagande sur le Brésil que les évènements de 1924 empêcheront.

La révolution culturelle avait eu lieu à São Paulo en février 1922. Paulo Prado en avait sonné le coup d'envoi, et les modernistes ne voulaient pas s'en tenir qu'à une Semaine d'agitation. Oswald en parle dans sa conférence à la Sorbonne sur "L'effort intellectuel du Brésil contemporain", une quinzaine de jours avant la visite du 28 mai chez Madame Duchâteau. Les modernistes connaissaient la poésie "ultra-réaliste" de ce sacré diable de Cendrars, poète de tous les fronts. Mário de Andrade, dans son article de 1924 (qui prend le relai de celui d'Oswald publié dans la presse un mois plus tôt) d'une profonde lucidité sur l'œuvre poétique de Cendrars qui venait de débarquer au Brésil, mais justement dubitatif sur la prose encore limitée du poète, écrit: "Cendrars surpreendeu e captou nos seus versos o grugrulho vasto do universo. Agora sim: inteiramente isento de retórica, pois que livre até de si mesmo e de seus próprios processos...". ${ }^{3}$ Un peu plus loin, dans un des plus beaux portraits qu'on ait sans doute jamais écrit sur Cendrars:

Fala o russo, o alemão, o italiano, o inglês, o português, o espanhol, etc. Deu um dos seus braços à França, na guerra. Tem um coração de criança. É simples e modesto como um trapista. Sociável e fino como un secretário de Legação brasileira sem, entretanto, preocupar-se com o corte dos smokings e das casacas. Poeta, tem o senso prático de um corretor de praça. Crítico, não tem os açaimos de escolas. Palestrador, deslumbra sem fazer frases nem ruídos. É simples e colorido e encanta, por que tem o que narrar. Viu. Sentiu. Viveu. Sua existência é ação e investigação.

Dans sa préface de 1967 aux poèmes Du monde entier, Paul Morand écrivait, et le répète plus d'une fois, que Cendrars "avait déjà tout vu" avant 1914, et que, "sorte de Tolstoï du transsibérien, ce huitième oncle", avait tout chanté.

Le Brésil, il le chantera jusqu'à son dernier souffle.

Tarsila et Oswald ont dans leur carton un projet avec les Ballets suédois, auquel participerait Heitor Villa-Lobos, et Cendrars leur propose de

3. EULÁLIO. A aventura brasileira de Blaise Cendrars, p. 157.

4. EUlÁlIO. A aventura brasileira de Blaise Cendrars, p. 161. D'origine suisse, Cendrars est naturalisé français en 1916.

5. MORAND. Préface. In: CENDRARS. Du monde entier (Poésies complètes 1912-1924), p. 9-12. 
rencontrer Rodolf de Maré, le fondateur des Ballets en 1920, mais malheureusement, leur proposition ne verra pas le jour.

Cendrars s'activera encore pour permettre l'exposition de Tarsila, Galerie Percier, rue de la Boétie à Paris, en juin 1926.

Nous allons essayer, dans cet article, de voir en quoi Le Lotissement $d u$ ciel se trouve profondément liée à la petite peinture de Cendrars offerte à Tarsila lors de cette première rencontre en 1923, même si à la rédaction de ce livre, les solides liens d'amitiés sont dénoués depuis de nombreuses années.

La petite peinture, une huile sur carton de 12 x 29 centimètres, s'est appelée depuis Torre Eiffel. ${ }^{7}$ Cendrars l'avait peinte en 1913, une jambe cassée, immobilisé dans une chambre d'hôtel dumasienne avec vue sur la Tour Eiffel, les Invalides, le Panthéon et le Sacré-Cour (tout à la fois! nous soulignons). Il nous en parle: Robert "Delaunay venait presque tous les jours me tenir compagnie. Il était toujours hanté par la Tour et la vue que l'on avait de ma fenêtre l'attirait beaucoup. Souvent il faisait des croquis ou apportait sa boîte de couleurs." Cendrars en profite, il se met à peindre, une peinture par jour, vingt-neuf au total. Robert Delaunay avait commencé à peindre en 1909 sa fameuse série des tours Eiffel, une trentaine de toiles représentant sous des angles inédits la "Grande Dame de fer". Il poursuivait ses recherches "constructivistes", dites aussi "déconstructivistes", avec la série des Fenêtres.

Si l'on regarde la Torre Eiffel de Cendrars, ce qu'il a couché avec les tubes de Delaunay sur le premier carton qui a dû lui tomber sous la main, un carton vagabundo, on distingue des tons dominants orangés (jaunes, orangés, ors et rouges), et des tons dominants bleus (bleus, violets et indigos). Difficile de dire quel jeu de dominante passe en premier plan. À mieux regarder, on croit voir des reflets de la tour Eiffel sur la Seine qui coule entre ses deux rives, à l'horizontale.

6. Cf. AMARAL. Blaise cendrars no Brasil e os modernistas, p. 143-146. Cendrars invite Tarsila et Oswald en automne 1923 à assister à La Création du monde au Théâtre des Champs-Élysées, par la Compagnie des Ballets suédois, sur son propre livret inspiré du poème "Fân", avec le danseur et chorégraphe Jean Börlin, une musique de Darius Milhaud, qui avait connu le Brésil avant Cendrars, enfin des décors et costumes de Fernand Léger.

7. On peut la voir à la Pinacothèque de l'État de São Paulo ou sinon sur la quatrième de couverture de Blaise Cendrars no Brasil e os modernistas.

8. CENDRARS. "La Tour Eiffel" dans "Peintres" dans Aujourd'bui. In: CENDRARS. Euvres complètes, v. 11, p. 75. 
Seule la couleur et ses contrastes déliminent, dessinent. Du post-impressionisme, plus proche des Nabis et du fameux petit Talisman de Paul Sérusier, de 1888, que de tous ces amis cubistes d'alors, synthétiques comme Picasso, orphiques comme Delaunay ou constructivistes comme Léger.

Cendrars peint et en clame la difficulté dans son poème "Journal", d'août 1913, le premier de ses Dix-neufs poèmes élastiques, une suite des "Pâques à New York", son poème inaugural. Cendrars préfère parler de peinture, de ses amis peintres, plutôt que de lui, peintre, dans un ensemble d'articles qui parâtront initialement dans la revue La Rose Rouge, de mai à août 1919, et regroupés ensuite par Cendrars en 1931 sous le titre "Peintres" dans Aujourd'bui.

Dans son troisième texte des "Peintres", sur l' "effritement" du cubisme et adressé à Albert Gleizes et Jean Metzinger, théoriciens d'un cubisme dit scientifique, la tour Eiffel apparâit comme modèle d'avenir: "Vis-à-vis de ce que sera la peinture constructive de demain, le cubisme théorique est comme le Trocadéro vis-à-vis de la Tour Eiffel: sans avenir, sans lendemain, sans utilisation possible." Dans son septième texte des "Peintres", consacré à son ami Delaunay, il écrit :"Mais on ne peut parler de ces peintres et de leur lyrisme intense, universel et coloré, pour lequel Guillaume Apollinaire avait trouvé le joli nom d'OOrphisme' sans parler de Robert Delaunay, le peintre de la tour Eiffel, l'inventeur du Simultané." Et de continuer son commentaire par l'inclusion d'un texte publié en Allemagne en mars 1914, ${ }^{12}$ qu'il réintitule Le Contraste simultané. Nous y reviendrons. Vient ensuite le huitième texte des "Peintres", cinématographique, sur Léopold Survage, son maître en peinture, puis un neuvième texte sur son ami Marc Chagall. Enfin, avant de "prendre congé des peintres", on va trouver le texte intitulé "La Tour Eiffel”, dédié à Madame Sonia Delaunay dans lequel Cendrars va

9. Aujourd'hui, paru chez Grasset en 1931, fait partie du volume 11 des Euvres complètes, publiés chez Denoël en 2005. Claude Leroy compare Aujourd'hui au Manifeste du Surréalisme de André Breton, de 1924.

10. CENDRARS. Aujourd'hui. In: CENDRARS. Euvres complètes, v. 11, p. 60.

11. CENDRARS. Aujourd'hui. In: CENDRARS. Euvres complètes, v. 11, p. 69. Après l'exposition cubiste de la Section d'Or d'octobre 1912, galerie de Boétie, Guillaume Apollinaire parle du cubisme "orphique" de Delaunay, en référence à son poème "Orphée", de 1908.

12. Voir note 80, p. 489 du volume 11 des Euvres complètes. 
nous parler de Robert Delaunay et de la tour Eiffel. ${ }^{13}$ Cendrars reviendra sur Delaunay et cette époque dans "La Tour Eiffel Sidérale", la troisième partie du Lotissement $d u$ ciel, qu'il dédie à la plus belle des Paulistes du monde, Tarsila do Amaral.

Cendrars avait rencontré et assez vite sympathisé avec le couple Delaunay dans une soirée organisée par Apollinaire. Ils venaient de faire connaissance quelques jours avant, le 10 octobre 1912, au Salon de la Section d'Or ${ }^{14}$ qu'Apollinaire animait et qui réunissait, galerie de la Boétie, tous les peintres cubistes du moment, mais salon duquel Delaunay avait décidé de se démarquer. Picabia et Duchamp s'y trouvent, Duchamp y présentant son $N u$ descendant un escalier. L'avant-garde parisienne en pleine ébullition, simultanément.

En 1912, Cendrars venait de rentrer de New York avec ses Pâques en poche et n'allait pas tarder à spéculer avec Sonia en russe (qu'il parlait après sa fugue sur le transsibérien) sur un projet qui aurait eu la taille de la tour Eiffel, projet qui deviendra la fameuse Prose du transsibérien et de la Petite Jehanne de France, le premier livre "simultané", dont l'édition, pages et exemplaires réunis, devaient dépasser les $300 \mathrm{~m}$. Cendrars et Apollinaire se lient d'amitié, mais Apollinaire semble ne pas vouloir reconnaître - comme beaucoup - le magnétisme de ce métèque de Cendrars. "Zone", le poème qui ouvre Alcools, son fameux recueil de 1913, aurait été placé en tête et la ponctuation supprimée après la lecture de $L a$ Prose du Transsibérien. "Zone" commence comme ceci:

À la fin tu es las de ce monde ancien

Bergère ô tour Eiffel le troupeau des ponts bêle ce matin

Tu en as assez de vivre dans l'antiquité grecque et romaine

Ici même les automobiles ont l'air d'êtres anciennes

La religion seule est restée toute neuve la religion

Est restée simple comme les hangars de Port-Aviation

$(\ldots)^{15}$

13. Ce texte est l'extrait d'une conférence faite le 12 juin 1924 à São Paulo, Brésil. CENDRARS. Aujourd'hui. In: CENDRARS. Euvres complètes, v. 11, p. $74-80$.

14. La Section d'Or ou Groupe de Puteaux, collectif de peintres, dont les frères Duchamp, et de critiques liés au cubisme orphique.

15. APOLlinaire. Alcools, p. 7. 
Et Cendrars, dans "Tour", son deuxième poème "élastique”, daté d'août 1913:

(...)

O Tour Eiffel! (...)

Gong tam-tam zanzibar bête de la jungle rayon-X express bistouri symphonie

Tu es tout

Tour

Dieu antique

Bête moderne

Spectre solaire

Sujet de mon poème

Tour

Tour du monde

Tour en mouvement ${ }^{16}$

Avant de voir comment la "bête moderne" revient trente-six ans plus tard dans Le Lotissement du ciel, reparlons peinture, nous concentrant sur nos orangés et nos bleus de la petite Torre Eiffel aux accents hermétistes de l'époque, symbolistes, disons simultanés.

Cendrars savait de ses amis peintres, ou même directement du grand chimiste Michel Eugène Chevreul, ${ }^{17}$ pour l'avoir sans doute lu, que l'orange est renforcé par le bleu, sa complémentaire, et vice-versa. Chevreul montra scientifiquement qu'une couleur n'existe pas en soi, mais qu'elle dépend de son environnement, qu'elle va se retrouver renforcée par sa complémentaire, son opposée sur le cercle chromatique. Il est amusant de noter, et cela n'est pas sans relation avec Le Lotissement du ciel, qu'à lire plus attentivement le titre du chapitre neuf du traité de Chevreul - "De l'application de la loi du contraste dans l'hypothèse

16. CENDRARS. Dix-neuf poèmes élastiques. In: CENDRARS. Du monde entier. Poésies complètes 1912-1924, p. 71-73.

17. Chevreul, directeur de la Manufacture des Gobelins dès 1813, s'attaque scientifiquement au problème de la couleur dont il vulgarise les résultats en 1839 dans De la loi du contraste simultané des couleurs, et de ses applications, qui s'adressait en premier aux peintres, bien que la première sollicitation avait été celle de ses étudiants teinturiers. Les Impressionnistes, les Pointillistes, les Divisionnistes et nombre de peintres à leur suite, eurent le temps d'y puiser consciencieusement. 
où le rouge, le jaune et le bleu sont les seules couleurs primitives, et l'orangé, le vert, l'indigo et le violet des couleurs composées" - on notera que le savant ajoute une couleur, en trop dans son exposé, l'indigo, teinture sur laquelle il avait déjà travaillé aux Gobelins. En effet, pour que les couleurs s'opposent diamétralement, il en faut six et non sept. La complémentaire du rouge, c'est le vert; du jaune, le violet (ou, et non pas "et", l'indigo qui pourrait le remplacer, mais ne serait pas à sa place sur le cercle), enfin la complémentaire du bleu, c'est l'orange. On l'apprend même aujourd'hui à l'école, où l'on ne parle pas d'indigo; qui pourrait affirmer qu'il s'agit d'un violet, d'un bleu, d'un bleu violet foncé ou encore d'une autre couleur? À la fin D'Oultremer à Indigo, que Cendrars fait paraître en 1939, se trouve l'histoire humoristique d'un savant qui étudiera sa vie durant une fleur de l'Orénoque qui passe par toutes les couleurs et brille même la nuit "d'un bleu lumineux, couleur phosphorescente". Avant de conclure son récit, Cendrars pose la question suivante:

Mais, diantre, pourquoi est-ce que le fameux professeur a baptisé son lis sauvage septem colorata (...) ? Si je sais bien compter cela ne fait une série que de six couleurs. Pourquoi dit-il sept, parce qu'il y a sept ciels, sept couleurs dans l'arc-en-ciel, sept notes dans la gamme, que le sept est un chiffre mystique, ou est-ce simplement un distrait comme ce savant que cite Remy de Gourmont qui qualifia la coccinelle de coccinella septem punctata alors que tous les enfants savent que les points noirs sur le dos rouge des bêtes à bon Dieu vont toujours par paires : deux, quatre, six, huit, mais jamais, au grand jamais sept ?

Dans son recueil de poèmes Feuilles de route, que Cendrars fait publier en septembre 1924 au retour de son premier séjour au Brésil, et qu'il demande à Tarsila d'illustrer avec ses dessins de Rio de Janeiro et du Minas Gerais, se trouve "L'oiseau bleu". Cet oiseau bleu du poème est bleu, mais vert mordoré aussi, et noir, jaune doré, vermillon, vert, incarnat, bref "on le nomme le septicolore". ${ }^{19}$ Ce bel oiseau, Cendrars va nous en conter un peu plus dans Le Lotissement du ciel dont la première des trois parties du livre, "Le Jugement

18. CENDRARS. D'Oultremer à Indigo, p. 228. Quant à la référence à Remy de Gourmont, Mario de Andrade affirme: "Remy de Gourmont é a única influência aceita por Cendrars sobre sua personalidade." ANDRADE apud AMARAL. Blaise Cendrars no Brasil e os modernistas, p. 40.

19. CENDraRs. Au Cour du monde. Poésies complètes 1924-1929, p. 87. 
dernier", ${ }^{20}$ débute sur la fabuleuse ménagerie qu'il s'efforce de ramener du paradis brésilien en août 1924, à bord du Gelria. Non sans l'aide précieuse de Gasperl, le charpentier, sorte de Noé de l'arche-paquebot, en vue de pouvoir offrir à ses amis ses soixante-sept ouistitis-lions (dorés) et à "la petite fille" Raymone surtout, que Cendrars "aimait plus que tout au monde" et à qui est dédié le récit, ses deux cent cinquante "sept-couleurs". Raymone n'en verra qu'un, et encore, qui devait malheureusement succomber très vite, nous dit Cendrars, "dans la lumière crue d'une ampoule électrique".

Ramener des Tropiques, pour commencer, un tamanoir-drapeau (Myrmecophaga tridactyla), avec les ouistitis et les sept-couleurs, s'avère très difficile, mais l'aventure ne manque pas d'humour; elle vient cependant en ouverture de ce dernier livre des mémoires de Cendrars, testamentaire. Le "post-scriptum pour les âmes sensibles" rappelle encore le souhait de finir dans un grand cuveau d'indigo, dans le ventre d'un grand cachalot. ${ }^{22}$

On ne saurait circonscrire le phénomème de la couleur dans un cercle, même si celui-ci est constitué d'une infinité de points, chacun pouvant représenter une couleur. Pour définir une couleur, il faut d'autres paramètres, comme la lumière, et on va alors vers le centre du cercle, vers le blanc, ou alors vers l'extérieur, le noir, mais l'inverse vaut tout aussi bien. Le troisième paramètre, après la couleur sur le cercle chromatique et la lumière, serait la saturation de la couleur ou son intensité: du moins saturé au plus saturé, un gris devient gris bleuté pour aller jusqu'à un bleu intense, un bleu perroquet par exemple. Et si la couleur change, comme pour l'étrange fleur de l'Orénoque, on a affaire au paramètre du temps. ${ }^{23}$ Sans parler encore d'un autre paramètre, qui serait celui de la profondeur. Et nous pourrions penser qu'avec tous ces paramètres, nous avons l'univers de la couleur sous la main.

20. Cendrars a biffé sur le manuscrit l'autre titre: "Les Sept-couleurs".

21. CENDRARS. Le Lotissement du ciel, p. 37-54.

22. CENDRARS. Le Lotissement $d u$ ciel, p.53-54. Le vieux professeur à la recherche de la fleur de l'Orénoque, dans le dernier récit de D'Oultremer à Indigo, se réunissait avec ses collègues de l'Université d'Upsala dans le ventre d'une grande baleine où se tenait leur club, dont le canapé rouge était très convoité.

23. La Quatrième Dimension est une notion en vogue avant la Grande Guerre. Cendrars en parle précisément dans sa conférence du 12 juin 1924 à São Paulo. CENDRARS. Aujourd'hui, p. 76. Cf. Jean Clair dans Marcel Duchamp ou le Grand Fictif, Paris: Galilée, 1975. 
Mais limitons notre réflexion à cette opposition sur le cercle chromatique de deux couleurs comme l'orange et le bleu, dans l'idée de montrer en quoi l'écriture de Cendrars, ambivalente, se trouve renforcée par le jeu des complémentaires.

Le bleu, dans D'Oultremer à Indigo, Claude Leroy en dresse rapidement une liste, non exhaustive bien sûr, pour montrer que la gamme des bleus et "l'ordre de la variation l'emporte sur celui des contrastes, pourtant chers au poète du Transsibérien." 24 Dans "La Tour Eiffel Sidérale", les bleus prennent le dessus - nous pourrions en dresser une liste tout aussi longue - par le simple fait que le récit se veut une "Rhapsodie de la Nuit" et qu'elle a pour décor la fazenda du "Morro Azul", la Montagne Bleue, sous l'enseigne d'une constellation rêvée et photographiée "dans l'immensité bleue", appelée Torre Eiffel Sideral par Oswaldo Padroso, son propriétaire-ermite touché lui aussi par l'amour. ${ }^{27}$

Et nos orangés? Sans doute faut-il les voir déclinés sous une couleur encore plus précieuse que les lapis-lazuli de Delaunay, dont la lueur vaut bien aux yeux des hommes le bleu ou l'indigo profond du ciel, mais venue cette fois-ci des entrailles de la terre: l'or. L'or dont va nous parler précisément Cendrars, y compris en note, dans "La Tour Eiffel Sidérale". La chronique de L'Or, Cendrars l'avait écrite en quelques semaines au retour de ce premier séjour au Brésil, sur les conseils de Paulo Prado. Il compte une nouvelle fois sur Tarsila pour illustrer $L$ 'Or, ce qui ne se fera pas. Toujours dans "La Tour Eiffel Sidérale", nous apprenons qu'il fait au "Morro Azul":

(...) l'apprentissage de mon métier de romancier, car c'est au retour de ce premier voyage dans la province de São Paulo que j'ai publié L'Or chez Grasset (...) une histoire merveilleuse que je me suis mis tout à

24. LEROY. Préface. In: CENDRARS. D'Oultremer à Indigo, p. XXV.

25. "Rhapsodies de la Nuit" est le sous-titre de "La Tour Eiffel Sidérale", en écho aux "Rhapsodies gitanes" de L'Homme foudroyé (1945).

26. CENDRARS. Le Lotissement du ciel, p. 425.

27. L'histoire d'Oswaldo Padroso (nom composé d'Oswald de Andrade et de Paulo Prado), de son vrai nom Luiz Bueno de la Miranda, prisonnier de son amour pour Sarah Bernhardt, n'est pas sans liens avec celle de Cendrars et Raymone. Sous l'œil bienveillant de l'amie Tarsila, à qui est dédié la rhapsodie, rappelons-le.

28. CENDRARS. Le Lotissement du ciel, p. 501-504. 
coup à élaguer et à dépouiller pour en faire une histoire vraie (...), qui fit je ne sais comment son tour du monde (...), écrit linéaire exactement le contraire du mode d'écriture polymère et polymorphe mais semblablement universel que j'emploie présentement pour tracer le portrait d'un somnambule. ${ }^{29}$

"L'OR est un leurre" nous confie-t-il aussi dans les premières pages de "La Tour Eiffel Sidérale". ${ }^{30}$ Et pourtant l'or resplendit tout au long de la belle nuit tropicale de la rhapsodie. Les orangés de la passion ("Mes peintures me font mal/Je suis trop passionné/tout est orangé") ${ }^{31}$ cèdent la place aux vieux ors clairs ou foncés des villes coloniales du Brésil, couleurs plus lumineuses, plus riches de l'expérience d'une vie, qui s'enflamment aux lueurs des bougies. Les phrases n'ont rien perdu de leur style télégraphique ou photographique d'alors, de leur ultramodernisme comme disait Tarsila. Courtes, rapides, elles viennent enchâssées dans de longues phrases sans fin, où l'inévitable point final, point de fuite, est reporté le plus loin possible, des phrases qui s'élèvent en spirale plus baroques les unes que les autres, de ce "merveilleux" Baroque tropical doré de tout l'or des Mines Générales, qui n'avait pas manqué d'enchanter Cendrars en 1924 en visite à Minas Gerais avec ses amis modernistes.

Le Lotissement du Ciel, avec ses trois parties - nous n'avons même pas parlé de la deuxième partie dédié à son fils, et dédicacée à sa petite amie la porteuse de pain, récit placé sous l'emblème totémique du colibri au vol arrière, comme le livre-testament: "son vol vibratile est un trait, une micassure, une étincelle, une braise ardente, une poussière au soleil, une étoile filante, une larme de diamant, un éclat..." ${ }^{32}-$, est un livre qui en aura surpris plus d'un, en contrepoint de L'Or. "Toute son aventure de créateur, par de multiples retours sur elle-même, s'y résume

29. CENDRARS. Le Lotissement du ciel, p. 429.

30. CENDRARS. Le Lotissement du ciel, p. 294.

31. Cendrars. Du monde entier. Poésies complètes 1912-1924, p. 69.

32. CENDRARS. Le Lotissement $d u$ ciel, p. 240. Soixante-treizième et plus longue séquence sans point autre que final de la seconde partie du Lotissement, intitulé "Le nouveau patron de l'aviation", dédiée à la petite amie de son fils Rémy, aviateur, mort au Maroc le 26 novembre 1945 en manœuvre. "C'est du cinéma" nous confie Cendrars fin de la vingt-sixième séquence, qui annonce la pesante hagiographie des saints volants. Une sacrée ambivalence! 
et s'y met en scène pour une sorte d'ultime représentation", écrit Leroy. ${ }^{33}$ Nous aimerions rajouter sous le signe de l'ambivalence. ${ }^{34}$ Cette ambivalence qui réunit les contraires plutôt qu'elle ne les oppose, comme dans le nom même de Blaise Cendrars, adopté à New York en 1912, la braise du phénix sous les cendres, ou encore le Brésil, son "Utopialand", un non lieu (utopia) qui est bel et bien un lieu (land), le Brésil, et non une île sans coordonnées, malgré les indices. ${ }^{36}$

Il serait tentant de montrer cette ambivalence des ombres et des lumières dont la fleur de l'Orénoque pourrait devenir l'emblème, de par sa couleur phosphorescente qui brille dans l'obscurité, ou ce "Diable" de Sac à Charbon ${ }^{37}$ du ciel austral, nébuleuse sombre que les astronomes connaissent bien mais de l'existence duquel Cendrars nous fait douter pour mieux nous faire croire à la force d'un mythe, ce Sac à Charbon voisin de la Croix du Sud tout comme sa sœur jumelle, la Boîte à Bijoux, cette autre curiosité stellaire (Cappa Crucis ou NGC 4755, dont les étoiles brillent d'éclats bleu pâle et orangé), un peu moins connue que son frère jumeau et dont Cendrars semble ne pas vouloir parler comme on cache un trésor. Il préfère cependant évoquer une autre boîte à bijoux, celle dans laquelle il se retrouve enfermée entre 1904 et 1907, comme apprenti joaillier (et poète) chez Leuba, un des plus riches joailliers de Saint-Pétersbourg d'alors. Les prémisses de la révolution de 1917 agite déjà la Russie tsariste; la toute-puissante Okhrana, la police politique du tsar sévit au dehors. "Dans la chambre noire de l'imagination",

\section{LEROY. Préface. In: CENDRARS. Le Lotissement du ciel, p. 7.}

34. Ne confondons pas ambivalence et ambiguité. Selon notre Petit Robert, est ambigu ce "qui présente deux ou plusieurs sens possibles, dont l'impression est incertaine". Dans une deuxième acception, philosophique, ce qui est "mal déterminé, qui semble participer à des natures contraires et appeler des jugements contradictoires." Du côté de l'ambivalence, on lit ceci: "caractère de ce qui se présente sous deux aspects, sans qu'il y ait nécessairement opposition ou ambiguité." Ce qui nous intéresse. Le binaire, lui est composé en tout et pour tout de deux éléments. La dualité fait coexister ces deux éléments, comme la bi-polarité qui les opposerait.

35. CENDRARS. Trop c'est trop. In: CENDRARS. Euvres complètes, v. 11, p. 305 .

36. Il serait intéressant de faire le lien entre l'île d'Utopia de Thomas More, Amerigo Vespucci et Caramurù dont nous parle Cendrars dans la deuxième partie du Brésil. Euvres complètes, vol. 11.

37. CENDRARS. Le Lotissement du ciel, p. 319.

38. CENDRARS. Le Lotissement du ciel, p. 399. 
Cendrars peint, avant de se servir des tubes de Delaunay: "Ce jour-là, je recomposai avec mes pierres la plus extraordinaire des compositions de Fouquet et la plus grandiose, et j'allumai toutes les bougies. C'était son chef d'œuvre: La Trinité dans sa Gloire, l'apothéose de DIEU, à ce jour la plus merveilleuse des galaxies (...)." ${ }^{39}$ Un peu avant:

(...) l'emploi des pierres précieuses dont je me servais en contrefaçon schématique de ses célèbres enluminures me permettait d'égaler en brillant et par opposition et même de dépasser spirituellement dans mes mosaïques à grande échelle, de la dimension de ma table de tri, car les perles, les rubis, les saphirs, les émeraudes, les topazes, les améthystes, les béryls, les diamants chantent en contraste simultané comme en cour (...). ${ }^{40}$

Dans La Trinité et tous les saints, de Jean Fouquet, la Trinité, dans sa gloire d'où émane une lumière jaune dorée éblouissante, se tourne vers la Vierge Marie, comme toute l'assemblée. Viennent border la gloire d'abord une couronne d'anges oranges, les Séraphins, les "brûlants", et ensuite, en contraste bien marqué, comme à l'infini, une couronne d'anges bleu céleste, les Chérubins, gardiens du royaume céleste, garants du mouvement perpétuel, cher à Cendrars. Le récit laisse place à une réflexion sur le suicide, sur lequel il revient dans un Prière d'insérer, qu'il écrit pour la première édition de 1949 du Lotissement. ${ }^{41}$

Cette symbologie ne nous intéresse ici que pour rapprocher la petite peinture de 1913, offerte à Tarsila em 1923, on s'en souvient, et cette mosaïque qu'il peint avec les mots lorsqu'il rédige ses mémoires dans sa retraite aixoise, dans sa cuisine sans feu, volontairement confiné durant quatre ans, écrasé par le poids du monde, ce depuis la Débacle de mai $1940{ }^{42}$

39. CENDRARS. Le Lotissement du ciel, p. 420. S'agit-il de La trinité et tous les saints, plutôt que de La Trinité dans sa gloire? La Trinité et tous les saints, Heures d'Étienne Chevalier, enluminées par Jean Fouquet, se trouve au Musée Condé, à Chantilly.

40. CENDRARS. Le Lotissement du ciel, p. 419-420.

41. CENDRARS. Le Lotissement du ciel, p. 581-582.

42. Les ébauches du Lotissement du ciel, considéré comme le quatrième et dernier tome des ses mémoires, ont commencé en 1928. Cendrars s'y plonge pendant la Seconde Guerre mondiale, à Aix-en-Provence, avec la profondeur du recul quant au Brésil et dans le plus grand dénuement d'un exil forçé. Les Allemands n'ont pas aimé son Chez l'armée anglaise (1940) et sa couverture bleu blanc rouge, la Gestapo est à ses trousses. 
Dans "Le Contraste simultané" qu'il écrit sur "les intentions" de Delaunay, il insiste sur la profondeur: "Le contraste n'est pas un noir et un blanc, un contraire, une dissemblance. Le contraste est une ressemblance. (...) C'est par contraste que les astres et les coeurs gravitent. C'est le contraste qui fait leur profondeur. Le contraste est profondeur. Forme." ${ }^{43}$

La profondeur de l'écriture de Cendrars, telle que nous la percevons, nous l'entendons dans cette lecture, pourrait se résumer à ce jeu ambivalent, perpétuel des contrastes colorés. Nous avons parlé de l'orange et du bleu mais cela vaudrait la peine - et le plaisir - de parler du rouge: la braise, le sang (la blessure de la guerre, si présente dans l'œuvre de Cendars) et du vert (le renouveau brésilien...). Ambivalence des complémentaires dans laquelle le superficiel du monde extérieur affleure les abymes du monde intérieur, les histoires sacrées se mêlent aux mythologies profanes, la simplicité ou l'humour du récit épousent la complexité de l'œuvre ou sa gravité, enfin, jeu incessant d'apparentes oppositions qui nous emmènent au bout des mondes de Blaise Cendrars, en poésie et en prose, mondes ou univers d'autant plus vrais d'ailleurs qu'il est peu vraisemblable qu'ils soient faux. Toujours entre écriture et aventure, quoiqu'on en dise, simultanément.

La première édition du Lotissement du ciel comprend ce Prière d'insérer où Cendrars recommande, et cela vaudra aussi comme notre conclusion:

(...) la vie n'est pas un dilemne et qu'entre les deux idéologies contraires entre lesquelles on les [jeunes gens] somme d'opter, il y a la vie, la vie, avec ses contradictions bouleversantes et miraculeuses, la vie et ses possibilités illimitées, ses absurdités (...). Il n’y a pas d'autre choix possible. Vivre...". ${ }^{44}$

Dans la sixième des cent cinq séquences de Saint Joseph de Cupertino de la deuxième partie du Lotissement - une séquence cinématographique émouvante! - Blaise engueule son fils Rémy: "Quand on a la chance de sortir une aussi jolie fille que ta porteuse de pain, que diable, on lui paye un bain et une liquette! ${ }^{45}$ La petite porteuse de pain au chômage rencontrée au cinéma par

43. CENDRARS. Aujourd'hui. In: CENDRARS. Euvres complètes, v. 11, p. 69.

44. CENDRARS. Le Lotissement du ciel, p. 581-582. Le monde est alors dit "bi-polaire" (Est/Ouest). L'existencialisme bat son plein.

45. CENDRARS. Le Lotissement du ciel, p. 79. 
Rémy n'a pas d'autre nom, un comble pour Cendrars! Cette petite porteuse de pain, une grande fille, est crasseuse, affamée et vulgaire, luronne, gourmande et belle, elle n'a pas vingt ans, elle est fougueuse, ardente, pétulante, "du Picasso. Du pire", ${ }^{46}$ nature enfin, amusante... Si l'on cherche dans Le Lotissement une allégorie de la vie, on devrait pouvoir en trouver plus d'une. Mais de plus belles et de plus vivantes que la petite porteuse de pain, sans doute pas.

En tête de "La Tour Eiffel Sidérale" qui clôt ses mémoires, Cendrars salue Tarsila, comment l'oublier, la plus belle et la plus vraie des Paulistes du monde.

Nous avons parlé de couleur, d'ambivalence. Cendrars parle de cour - laissons-lui le dernier mot - quand il commente lui-même son Dan Yack(1946), la réunion en un seul volume du Plan de l'Aiguille et des Confessions de Dan Yack (1929), livre utopique et contre-utopique à la fois: "Systole, diastole: les deux pôles de l'existence; outside-in, inside-out: les deux temps du mouvement mécanique; contraction, dilatation: la respiration de l'univers, le principe de la vie: l'Homme..."

Cendrar's orange and blue, simultaneously

Abstract: During their first meeting in Paris in 1923, Blaise Cendrars gave to Tarsila do Amaral a small oil painting on card entitled Torre Eiffel, one of the very few he ever painted ten years before with his friend Robert Delaunay's paints. Twenty years after his last stay in Brazil, Cendrars would dedicate to Tarsila the last story of his Memoirs, taking place in the fazenda "Morro Azul", belonging to some Oswaldo Padroso, the discoverer of a new constellation: the "Sideral Eiffel Tower", which inspired the title of that tale. The oranges of passion and the blues of introspection would mix throughout his life, in both ambivalence and simultaneity of contrasts, just in the same way as his adventures and his writing. Thus the poet would for ever sing the ambers still glowing under the ashes.

Keywords: Blaise Cendrars, Tarsila do Amaral, Eiffel Tower, Contrasts, Simultaneity, Ambivalence.

46. CENDRARS. Le Lotissement du ciel, p. 71.

47. CENDRARS apud LEROY. Préface. In: CENDRARS. Euvres complètes, v. 4 , p. X. 


$$
\text { Références }
$$

AMARAL, Aracy. Artes plásticas na Semana de 22. São Paulo: Perspectiva, 1970.

AMARAL, Aracy. Blaise Cendrars no Brasil e os modernistas. 2. ed. São Paulo: Editora 34, 1997.

APOLLINAIRE, Guillaume. Alcools. Paris: Gallimard, 1920.

CENDRARS, Blaise. Du monde entier. Poésies complètes 1912-1924. Paris: Gallimard, 1967.

CENDRARS, Blaise. Au cœur du monde. Poésies complètes 1924-1929. Paris: Gallimard, 1968.

CENDRARS, Miriam. Blaise Cendrars. L'or d'un poète. Paris: Gallimard, 1996.

CENDRARS, Blaise. Le Lotissement du ciel. Paris: Gallimard, 1996.

CENDRARS, Blaise. D'Oultremer à Indigo. Paris: Gallimard, 1998.

CENDRARS, Blaise. Dan Yack. In: Euvres complètes. Paris: Denoël, 2002. v. 4.

CENDRARS, Blaise. Aujourd'hui suivi de Jéroboam et La Sirène, Sous le signe de François Villon, Le Brésil et Trop c'est trop. In: . Euvres complètes. Paris: Denoël, 2005. v. 11.

CENDRARS, Blaise. O loteamento do céu. São Paulo: Companhia das Letras, 2009.

CHEVREUl, Michel Eugène. De la loi du contraste simultané des couleurs, et de ses applications. Paris: Pitois-Levrault, 1839.

EULÁLIO, Alexandre. A aventura brasileira de Blaise Cendrars. São Paulo: Instituto Nacional do Livro, 1978.

FREITAS, Maria Teresa de; LEROY, Claude (sous la direction de). Brésil. L'utopialand de Blaise Cendrars. Paris: L'Harmattan, 1998.

LEROY, Claude. Préface. In: CENDRARS. D'Oultremer à Indigo. Paris: Gallimard, 1998. p. I-XXVII. 